\title{
Abbreviations Used in the Text, Notes and References
}

NAMES OF ARCHIVES, LIBRARIES AND

COLLECTIONS OF DOCUMENTS USED

AKV Arkhiv kniazia Vorontsova

NLS National Library of Scotland, Edinburgh

RGADA Rossiiskii gosudarstvennyi arkhiv drevnikh aktov, Moscow

RGALI Rossiiskii gosudarstvennyi arkhiv literatury i iskusstva, Moscow

RGB Rossiiskaia gosudarstvennaia biblioteka, Moscow

RGIA Rossiiskii gosudarstvennyi istoricheskii arkhiv, St Petersburg

RIA Royal Irish Academy, Dublin

SIRIO Sbornik imperatorskogo russkogo istoricheskogo obshchestva

OTHER ABBREVIATIONS USED IN THIS VOLUME

d. delo (dossier, file)

ed. khr. edinitsa khraneniia (individual file)

f. fond (collection)

fol. folio (list in Russian)

Fr. French

k. $\quad$ karton (carton)

op. opis' (inventory)

OR Otdel rukopisei (manuscripts department)

r. recto

razd. $\quad$ razdel (division)

v. verso 